\title{
Pressure-induced orbital-selective metal from the Mott insulator $\mathrm{BaFe}_{2} \mathrm{Se}_{3}$
}

\author{
L. Craco ${ }^{1}$ and S. Leoni ${ }^{2, *}$ \\ ${ }^{1}$ Instituto de Física, Universidade Federal de Mato Grosso, 78060-900 Cuiabá, MT, Brazil \\ ${ }^{2}$ School of Chemistry, Cardiff University, Cardiff CF10 3AT, United Kingdom
}

(Received 28 November 2018; revised manuscript received 6 April 2020; accepted 27 May 2020;

published 9 June 2020)

\begin{abstract}
A general understanding of the mechanism underlying the pressure-induced Mott insulator-metal transition in strongly correlated materials is still lacking. Here we explore the pressure-induced electronic reconstruction in $\mathrm{BaFe}_{2} \mathrm{Se}_{3}$, a potential two-leg ladder system for unconventional (non-BCS) superconductivity. We stress the importance of multiorbital Coulomb interactions in concert with first-principles band-structure calculations for a consistent understanding of its intrinsic Mott-Hubbard insulating state both at ambient and under pressure. We elucidate the nature of pressure-induced insulator-metal transition seen in experiment, showing that it is driven by bandwidth broadening under pressure. We reveal an orbital-selective electronic state where Mott localized and itinerant electrons coexist in compressed $\mathrm{BaFe}_{2} \mathrm{Se}_{3}$, which incorporates orbital-resolved scattering rates and renormalization factors hidden in the normal state at high pressures.
\end{abstract}

DOI: 10.1103/PhysRevB.101.245133

\section{INTRODUCTION}

Orbital-selective charge and spin fluctuations in Fe-based superconductors are now recognized to represent a complex many-body problem of fundamental importance, and its understanding and implications has attracted much in condensed-matter and material physics community in recent years $[1,2]$. The majority of the Fe-based superconductors comprise a two-dimensional $\mathrm{Fe}$ square lattice tetrahedrally coordinated by pnictogens or chalcogens and display metallic, bad-metallic, and insulating normal states arising from the interplay among structural, orbital, charge, and spin degrees of freedom. Magnetic and electronic excitations have been extensively studied since their discovery in 2008 [3], but a unified description of many-particle correlation effects on electrons occupying all active Fe $3 d$ orbitals remains a challenging theoretical task [4].

Most of the parent Fe superconducting compounds show tetragonal-to-orthorhombic structural changes accompanied by para-to-antiferromagnetic phase transition at low temperatures $(T)$, typically exhibiting striped-type antiferromagnetic ordering [5]. According to theoretical analyses based on multiband models with hole and electron Fermi pockets, the striped-type magnetic ordering is stabilized by Fermi-surface nesting, and the associated magnetic (or spin) fluctuations are believed to induce unconventional high- $T_{c}$ superconductivity [6]. However, it has also been found that some Febased superconductors show significant deviations from the Fermi-surface nesting mechanism. Among the best-known examples are the alkaline $\mathrm{Fe}$ selenides, $\mathrm{K}_{1-x} \mathrm{Fe}_{2-y} \mathrm{Se}_{2}$, where superconductivity appears at the border of Mott localizationdelocalization transition [7]. Importantly, pressure-induced Mott insulator-to-metal transition followed by superconductivity has also been observed in the Fe ladder superconductor

*LeoniS@ cardiff.ac.uk
$\mathrm{BaFe}_{2} \mathrm{~S}_{3}$ [8]. This widens the understanding of electronic and magnetic instabilities [9] as well as of different possible pairing mechanisms [10] in Fe superconductors.

Whether unconventional superconductivity is intrinsic to Fe ladder compounds is still under debate, with experimental studies at high pressures displaying apparently conflicting results [11-13]. Particularly interesting in this context is the two-leg ladder $\mathrm{BaFe}_{2} \mathrm{Se}_{3}$ compound, where a striking experimental study suggests that superconductivity can be induced within a pressure range between 10.2 and $15 \mathrm{GPa}$ [11]. At ambient and low-pressure conditions the resistivity of pure $[11,14]$ and doped $[12,15,16] \mathrm{BaFe}_{2} \mathrm{Se}_{3}$ shows clear insulating behavior with a thermal activation energy close to $0.178 \mathrm{eV}$ above $170 \mathrm{~K}$ [14]. As expected in strongly correlated materials under extreme pressure conditions [17], the resistivity of $\mathrm{BaFe}_{2} \mathrm{Se}_{3}$ gradually decreases under compression and a pressure-induced Mott insulator-metal transition sets in between 8.8 and 10.2 GPa [11]. Albeit not clearly seen in recent experiments [12,13], the electrical resistivity of Ref. [11] shows metallic behavior up to $250 \mathrm{~K}$ above $10.2 \mathrm{GPa}$.

The application of pressure often induces strong manyparticle reconstruction of Mott localized electrons [17-19]. Therefore, an understanding of the hidden mechanism underlying the pressure-induced insulator-to-metal transition in $\mathrm{BaFe}_{2} \mathrm{Se}_{3}$ requires a precise description of the pressure evolution of its electronic structure. The challenges to microscopically explore the multiorbital (MO) electronic structure of $\mathrm{BaFe}_{2} \mathrm{Se}_{3}$ are as follows: What interaction strength determines the insulating state at ambient and low-pressure conditions? How is metallicity induced in a system, which is expected to be in close proximity to Mott localization? In this work we answer these questions, providing a microscopic description of electronic transport seen in the insulating and metallic phases of normal and compressed $\mathrm{BaFe}_{2} \mathrm{Se}_{3}$. In our modeling we assume that the observed insulator-metal transition is neither characterized by a structural distortion transition nor by the collapse of electron-electron interactions, but 


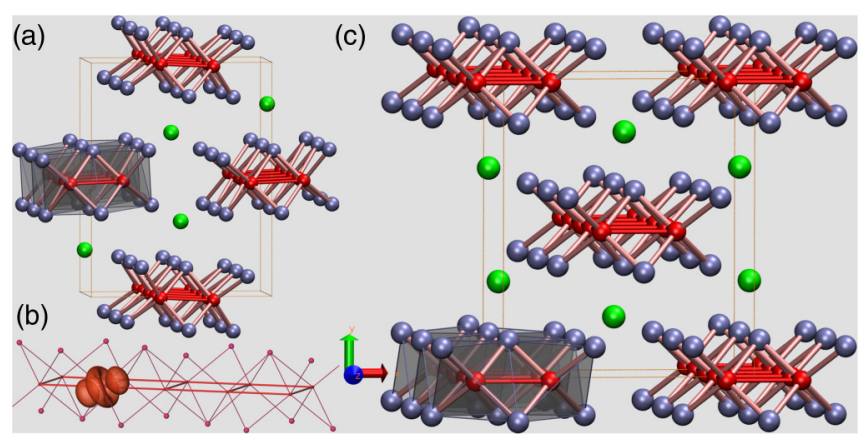

FIG. 1. Pressure-dependent polymorphism of $\mathrm{BaFe}_{2} \mathrm{Se}_{3}$ : (a) Ambient pressure crystal structure of $\mathrm{BaFe}_{2} \mathrm{Se}_{3}$ in the Pnma space group (Fe: red; Se: purple; Ba: green). Fe-Fe ladders are shown in red. One set of $\mathrm{Fe}-\mathrm{Se}$ tetrahedra is shown within a single ladder only (transparent gray polyhedra). (b) The electron density of the ground state orbital $3 z^{2}-r^{2}$ is shown as isosurface within a single Fe ladder. The $3 z^{2}-r^{2}$ orbital axis is close to the Fe-Fe ladder direction, $z$. (c) High-pressure $(12.7 \mathrm{GPa})$ polymorph, $\mathrm{Cmcm}$. The $z$ direction is chosen along the Fe ladders in all snapshots.

it is shown to be bandwidth-controlled Mott transition [8,19]. We reveal an orbital-selective (OS) phenomenon where Mott localized (with $x y, 3 z^{2}-r^{2}$ orbital character) and quasicoherent metallic states (with $x z, y z, x^{2}-y^{2}$ orbital character) coexist in $\mathrm{BaFe}_{2} \mathrm{Se}_{3}$ at $12.7 \mathrm{GPa}$, where the superconducting anomaly becomes sharp in experiment [11].

$\mathrm{BaFe}_{2} \mathrm{Se}_{3}$ is an antiferromagnetic Mott insulator with a Néel temperature $T_{N} \approx 256 \mathrm{~K}$ [20]. At ambient pressure (AP) it adopts the $\mathrm{CsAg}_{2} \mathrm{I}_{3}$-type structure (Pnma space group) which can be viewed as distorted $\mathrm{BaFe}_{2} \mathrm{~S}_{3}(\mathrm{Cmcm}$ space group). Each unit cell has two Fe ladders along $z$ direction (see Fig. 1) which are built by edge-sharing $\mathrm{FeSe}_{4}$ tetrahedra $[12,14,21] . \mathrm{BaFe}_{2} \mathrm{Se}_{3}$ hosts a block magnetic order with its magnetic spin moments aligned perpendicular to the leg direction $[20,22]$, in contrast to the Fe-pnictide superconductors which usually exhibit stripe magnetic order. Similarly to $\mathrm{BaFe}_{2} \mathrm{~S}$ [23], it has been proposed that $\mathrm{BaFe}_{2} \mathrm{Se}_{3}$ forms an OS Mott phase, where localized and itinerant electrons coexist in the normal state $[20,22,24]$. By applying pressure, a structural transition is induced around $6 \mathrm{GPa}$, transforming the crystal into a higher-symmetry structure, similar to that of compressed $\mathrm{BaFe}_{2} \mathrm{~S}_{3}$ [25]. However, whether an OS metallic state can be induced and how it will evolve under pressure is still unknown in this material. Motivated thereby, in this work we provide a local density approximation plus dynamical mean-field theory (LDA+DMFT) [26] description to this problem, showing good theory-experiment agreement with ultraviolet photoemission spectroscopy (UPS) at ambient pressure [23] as well as with resistivity data for both normal and compressed structural phases of $\mathrm{BaFe}_{2} \mathrm{Se}_{3}$ [11].

\section{THEORY AND RESULTS}

Using the experimentally determined crystal structure parameters at AP [27] we have performed LDA band-structure calculations within the linearized muffin-tin orbitals (LMTO) method in the atomic sphere approximation [28]. To obtain structural parameters for the $\mathrm{Cmcm}$ structure, we have

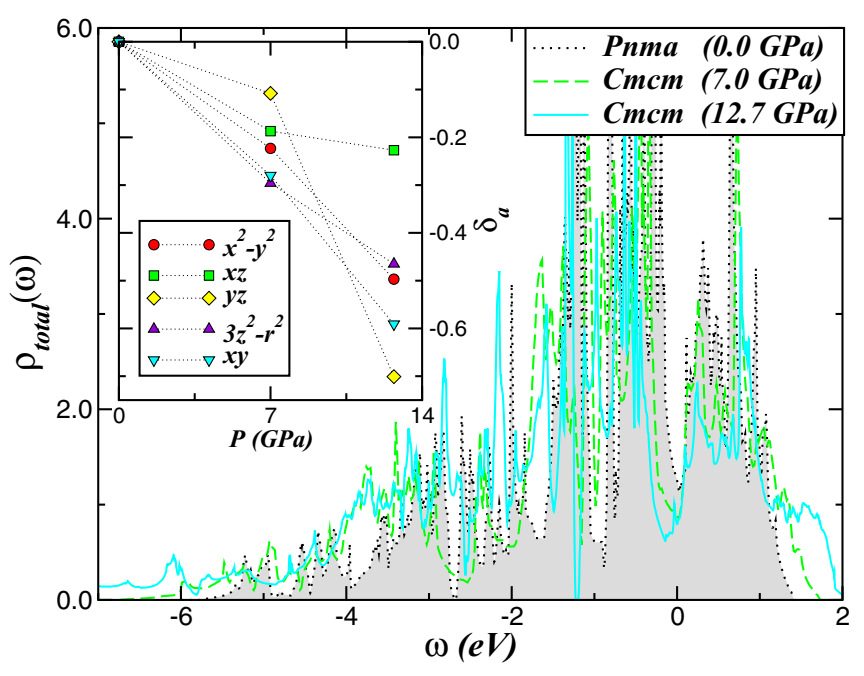

FIG. 2. Pressure evolution of the total LDA DOS for the Fe $3 d$ orbitals of paramagnetic $\mathrm{BaFe}_{2} \mathrm{Se}_{3}$. An important feature to be seen is the bandwidth broadening with increasing pressure. Inset shows the relative changes in the orbital-dependent crystal field splitting $\delta_{a} \equiv \delta_{a}(P)-\delta_{a}(\mathrm{AP}) \quad\left(a=x^{2}-y^{2}, 3 z^{2}-r^{2}, x z, y z, x y\right)$, between ambient (AP) and two high-pressure cases relevant to transport experiments [11]. This shows that strong, bare electronic reconstruction occurs in compressed Fe spin-ladder compounds.

performed additional LDA calculations by relaxing all atomic coordinates at experimental lattice constant values [29]. [Relaxed Wyckoff positions: $\mathrm{Fe} \quad(8 e) 0.14870 .00 .0$, $\mathrm{Ba} \quad(4 c) 0.00 .31590 .75, \quad \mathrm{Se}_{1}(4 c) 0.00 .87010 .75$, and $\mathrm{Se}_{2}(4 c) 0.00 .13$ 0.25.] Our LDA results in Fig. 2 confirm that the active electronic states in this Ba 123-spin-ladder compound are the $\mathrm{Fe} 3 d$ carriers, where all $3 d$ bands (see Fig. 4) have appreciable weight below and above the Fermi energy, $E_{F}=\omega=0$ [30]. As displayed in Fig. 2, the total density of states (DOS) shows metallic behavior at AP in contrast to the insulating state seen in transport $[11,12,14]$ and optical conductivity spectra [15]. This can be taken as an evidence that correlated band-structure calculations [26] are needed to unveil the one-particle spectra of normal and compressed $\mathrm{BaFe}_{2} \mathrm{Se}_{3}$.

It is noteworthy that the use of a localized basis has important technical advantages in order to reduce the number of nonzero short range electron-electron interaction terms [31]. Analogously to LDA+U calculations, we choose a projection local basis in which the Fe $3 d$ occupancy matrix is diagonal. However, due to the strong hybridization of $\mathrm{Fe} 3 d$ orbitals with Se $4 p$ orbitals [see LMTO band structure, Fig. 4(a)], the number of bands considered for the calculation of the occupation matrix, and therefore the projection band energy range, are critical factors. To illustrate this, we have constructed a tight-binding model based on Wannier functions [32], using 76 bands and as many Wannier projectors centered on Fe and Se atoms. To obtain this tight-binding model (see our result below), first-principles calculations for $\mathrm{BaFe}_{2} \mathrm{Se}_{3}$ at ambient pressure have been performed using $p w . x$ from the Quantum Espresso [33] distribution v6.4.1, using the PerdewZunger (LDA) exchange correlation functional. Pseudopotentials (PAW) from the PSLibrary [34] have been used. Cutoffs 

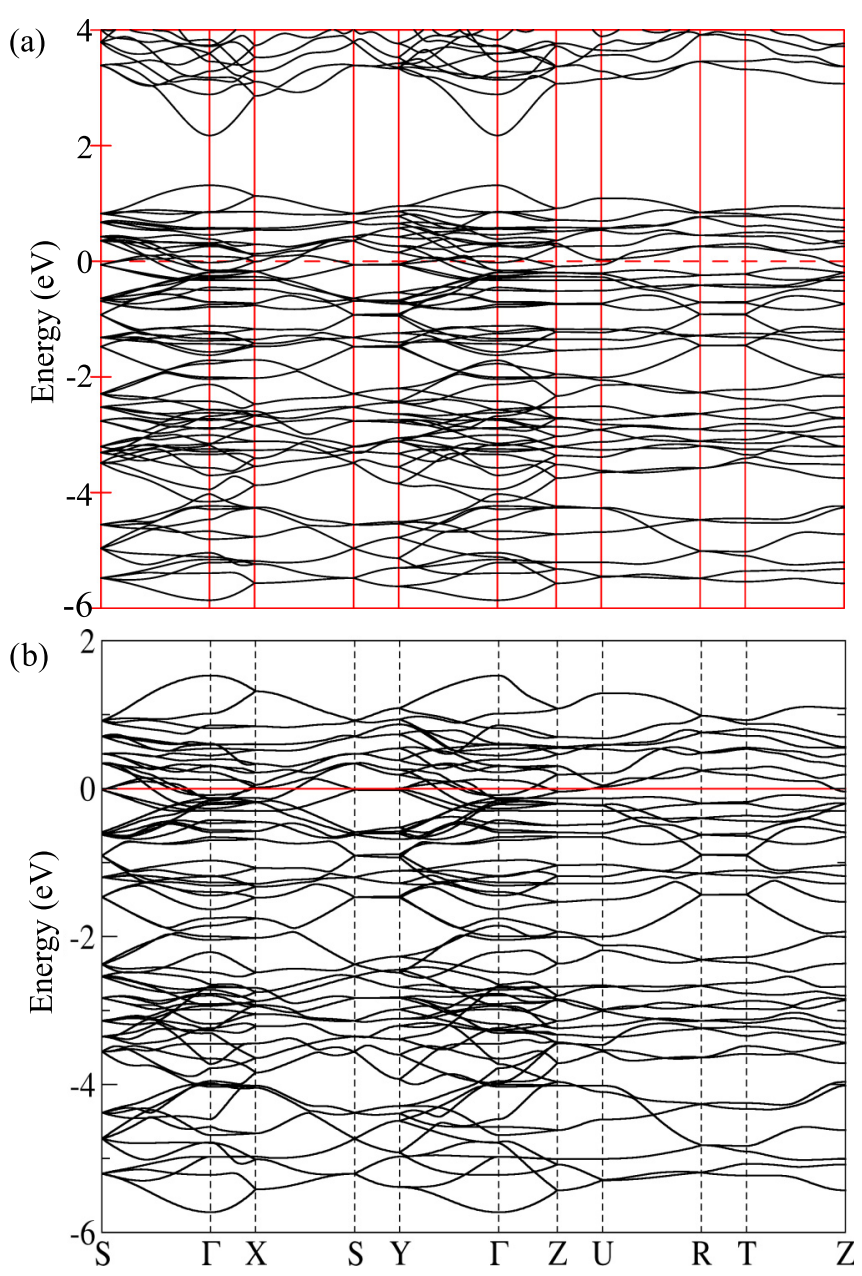

FIG. 3. Projected band structures of $\mathrm{BaFe}_{2} \mathrm{Se}_{3}$ at ambient pressure obtained from LMTO (a) and reconstructed (b) from maximally localized Wannier functions for the nonmagnetic state. In the latter, 76 bands are shown (Se $4 p$ and Fe $3 d$ ). Higher- and lower-lying bands were not considered. The Fermi level is at zero energy and it is shown with dashed (solid) lines. The band structure is more dispersive from $\Gamma$ to $Z$ than along other directions, which is consistent with the presence of quasi-one-dimensional ladders along the $k_{z}$ axis, in agreement with extant calculations for $\mathrm{RbFe}_{2} \mathrm{Te}_{3}$ and $\mathrm{BaFe}_{2} \mathrm{~S}_{3}$ [36].

of 70 and 800 Ry were chosen for the expansion of the wave functions and the charge density, respectively. The charge density was converged self-consistently to an error smaller than $1.0^{-8} \mathrm{meV}$. Maximally localized Wannier functions have been calculated with version 3.01 of the Wannier90 code. $\mathrm{Fe} 3 d$ and $\mathrm{Se} 4 p$ orbitals were chosen as initial projections for a total of 76 band in the energy range -6.0 to $2.0 \mathrm{eV}$. Lower and higher energy bands have been excluded. Convergence has been achieved when the total spread change was less than $1.0^{-12} \AA$ for 10 iterations. The resulting reconstructed band structure shown in Fig. 3(b) is in good agreement with extant calculations for the nonmagnetically ordered state of $\mathrm{BaFe}_{2} 2 \mathrm{Se}_{3}$ [35]. The choice of this energy range guarantees Fe localized, atomic-like Wannier functions, with the correct site symmetry. In the same spirit, in LMTO the occupation matrix is calculated for all $76 \mathrm{Fe}$ and Se bands, over their relevant energy range [ -5.9 to $1.6 \mathrm{eV}$, Fig. 3(a)]. This provides

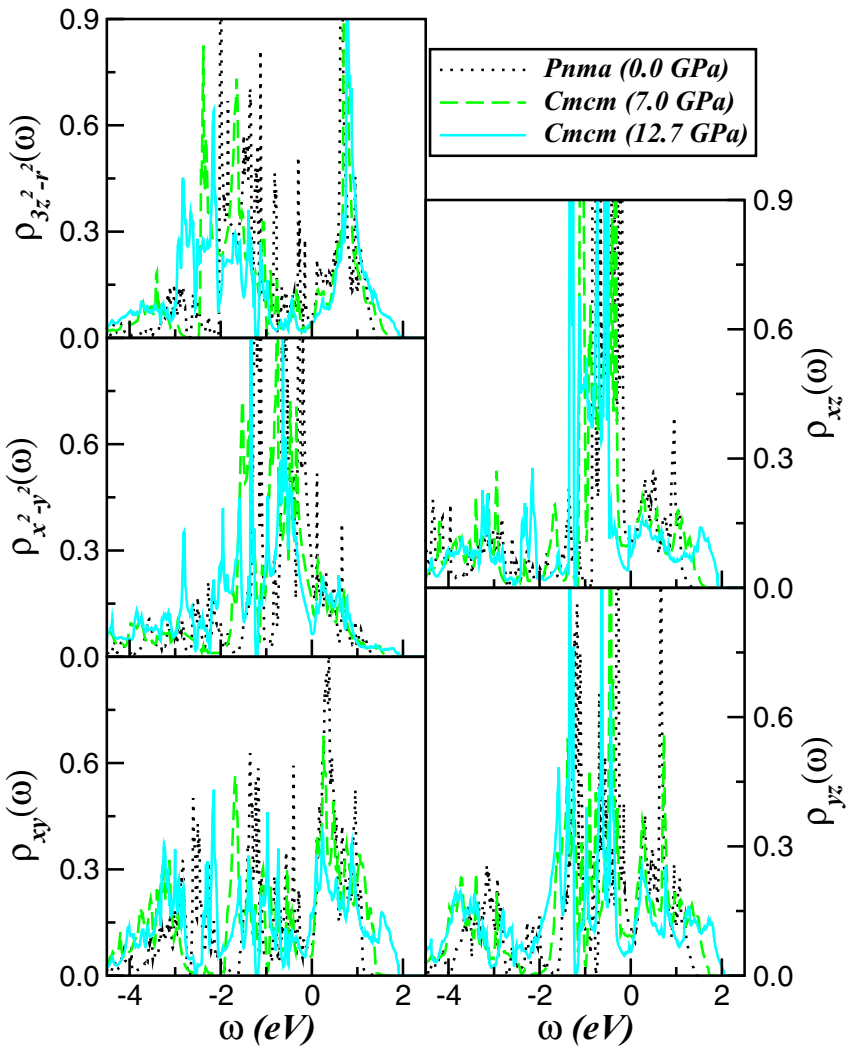

FIG. 4. Pressure evolution of the orbital-resolved LDA DOS for the $\mathrm{Fe} 3 d$ orbitals of $\mathrm{BaFe}_{2} \mathrm{Se}_{3}$. Important features to be seen is the bandwidth broadening and the emergence of an anisotropic, $\mathrm{V}$-shapelike electronic structure near $E_{F}$ with increasing pressure.

a robust strategy for the choice of an atomic-like basis in a situation of strong interband hybridization. The corresponding projected DOS are shown in Fig. 2.

Using the Wannier functions formalism we have also computed the bare (one-particle) Hamiltonian in the matrix form at ambient and the high pressure regime of the $\mathrm{Cmcm}$ phase considered in this work. In the basis used here, the on-site hopping matrix for $0.0 \mathrm{GPa}$ and $12.7 \mathrm{GPa}$ have, respectively, the form

$$
\hat{H}_{0}=\left[\begin{array}{lcccc}
-0.9847 & -0.0087 & -0.0789 & 0.0727 & 0.0223 \\
0.0087 & -0.8018 & 0.0219 & 0.0467 & -0.0320 \\
-0.0789 & 0.0219 & -0.9714 & 0.0846 & 0.0088 \\
0.0727 & 0.0467 & 0.0846 & -0.9143 & 0.0278 \\
0.0223 & -0.0320 & 0.0088 & 0.0278 & -0.8195
\end{array}\right],
$$

and

$$
\hat{H}_{0}=\left[\begin{array}{lllll}
-1.2781 & -0.0010 & 0.0099 & -0.0319 & -0.0016 \\
-0.0010 & -1.2774 & 0.0015 & -0.0021 & -0.0091 \\
0.0099 & 0.0015 & -1.3222 & -0.1496 & -0.0006 \\
-0.0319 & -0.0021 & -0.1495 & -1.3366 & 0.0006 \\
-0.0016 & -0.0091 & -0.0006 & 0.0006 & -1.3189
\end{array}\right] \text {, }
$$

with the orbital order representing $\hat{H}_{0}$ being $3 z^{2}-r^{2}, x z, y z$, $x^{2}-y^{2}$, and $x y$. As seen, the off-diagonal elements are close to zero in both cases, attesting our choice for the local axis used for the Wannier function projection. As expected all diagonal hopping elements increase with increasing pressure due to enhanced interorbital overlap, which is constituent with the bandwidth broadening with increasing pressure, see Fig. 2. Importantly, in the local basis which 
diagonalizes the one-particle density matrix, this residual interorbital one-electron overlap is zero, and so in the paramagnetic phase the many-particle Green's functions and selfenergies become diagonal in orbital and spin flavor [37].

Relevant to our study is the bandwidth broadening, which accompanies the pressure induced metals from Mott insulators [19]. As shown below the competition between oneparticle band broadening, which is enhanced by almost $2.0 \mathrm{eV}$ from AP to $12.7 \mathrm{GPa}$ and the local Coulomb repulsion $U$ in $\mathrm{BaFe}_{2} \mathrm{Se}_{3}$ generates an OS metallic state at high pressures. Similar bandwidth-control-type Mott transition has been proposed for $\mathrm{BaFe}_{2} \mathrm{~S}_{3}[8,38]$, and it therefore appears to be an intrinsic property of the $\mathrm{Fe}$ spin-ladder superconductors. In addition to band broadening also relevant for the Mott transition is the rearrangement of the $3 d$ electronic structure due to changes in the crystal field splitting under pressure [38]. This nontrivial structural phenomenon acts like an orbital field [39] which modifies the on-site orbital energies [10] or the center of gravity $\delta_{a}$ of the LDA $3 d$ manifold $a=x^{2}-$ $y^{2}, 3 z^{2}-r^{2}, x z, y z, x y$. As shown in the inset of Fig. 2, large changes in the orbital-dependent crystal field splitting [10] $\delta_{a}=\delta_{a}(P)-\delta_{a}(\mathrm{AP})$ takes place in $\mathrm{BaFe}_{2} \mathrm{Se}_{3}$ at the two pressure regimes considered in this work. As seen, particular strong reconstruction sets in within the $y z$ orbital at $12.7 \mathrm{GPa}$.

In order to get insights into the changes in the bare electronic structure of $\mathrm{BaFe}_{2} \mathrm{Se}_{3}$ from ambient to high pressures, in Fig. 4 we compare the corresponding orbital resolved LDA DOS. As seen, on compression we find substantial transfer of spectral weight, particularly at the valence band states below $E_{F}$. Albeit the overall shape of the LDA spectral functions shows only weak pressure and structural-phase dependence, our results exhibit clear increase in the bare bandwidth and subtle splitting of peaks on different orbitals. Interestingly and consistent with the pressure evolution in Fig. 2, our results reveal a nonnegligible reduction of the $\mathrm{Fe} 3 d$ spectral weight near $E_{F}$, particularly within the $x^{2}-y^{2}, x z, y z$ orbitals where the LDA DOS exhibit a monotonous increase of $\mathrm{V}$-shape-like electronic structure. Similarly to topological insulators and some Fe-based superconductors [40], this result can be taken as an evidence that anisotropic Dirac-fermion physics might be hidden in the bare electronic structure of $\mathrm{BaFe}_{2} \mathrm{Se}_{3}$ at pressure close to $12.7 \mathrm{GPa}$. Viewed in light of this result, our finding suggests interesting avenues for future studies.

The one-electron part of the $\mathrm{Fe} 3 d$ model Hamiltonian relevant for the $\mathrm{Fe}$ spin-ladder compounds is $H_{0}=$ $\sum_{\mathbf{k}, a, \sigma} \epsilon_{a}(\mathbf{k}) c_{\mathbf{k}, a, \sigma}^{\dagger} c_{\mathbf{k}, a, \sigma}$, where $a$ denotes its diagonalized $3 d$ orbitals and $\epsilon_{a}(\mathbf{k})$ is the corresponding band dispersion, which encodes details of the one-electron (LDA) band structure. These five $\mathrm{Fe} 3 d$ bands are the relevant one-particle inputs for MO-DMFT which generates a Mott-Hubbard insulating state at ambient and low-pressure conditions as shown below. The correlated many-body Hamiltonian considered for $\mathrm{BaFe}_{2} \mathrm{Se}_{3}$ reads

$$
\begin{aligned}
H_{\text {int }}= & U \sum_{i, a} n_{i, a, \uparrow} n_{i, a, \downarrow}+U^{\prime} \sum_{i, a \neq b} n_{i, a} n_{i, b} \\
& -J_{H} \sum_{i, a \neq b} \mathbf{S}_{i, a} \cdot \mathbf{S}_{i, b} .
\end{aligned}
$$

Here $U$ is the on-site Coulomb interaction, $U^{\prime}=U-2 J_{H}$ is the interorbital Coulomb interaction term, and $J_{H}$ is the

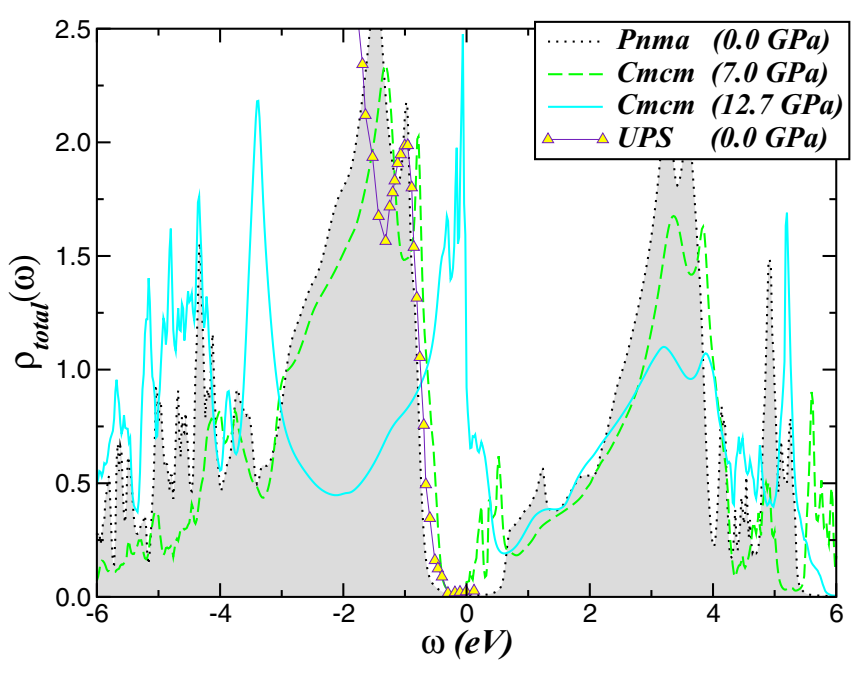

FIG. 5. Evolution of the LDA+DMFT $\left(U=4.7 \mathrm{eV}, J_{H}=\right.$ $0.7 \mathrm{eV}$ ) total spectral functions of normal and compressed $\mathrm{BaFe}_{2} \mathrm{Se}_{3}$. While all orbitals are Mott localized at ambient pressure, incoherentband states with $x y$ orbital character almost touch the Fermi energy $\left(E_{F}=\omega=0\right)$ at $P=7.0 \mathrm{GPa}$ due to pressure-induced selectiveorbital electronic reconstruction in $\mathrm{BaFe}_{2} \mathrm{Se}_{3}$. Noteworthy is the comparison between the LDA+DMFT DOS with ultraviolet photoemission spectra (UPS, triangles) [23], showing good quantitative theory-experiment agreement up $1.2 \mathrm{eV}$ binding energy. In particular, the low-energy line shape and the peak structure around $-1.0 \mathrm{eV}$ are accurately resolved by the insulating spectrum of $\mathrm{BaFe}_{2} \mathrm{Se}_{3}$. The solid line shows the energy dependence of the total $\mathrm{Fe} 3 d$ electronic structure which might be probed in future photoemission and inverse photoemission experiments of superconducting $\mathrm{BaFe}_{2} \mathrm{Se}_{2}$.

Hund's coupling. Consistent with earlier studies [41], we focus on the leading correlation effects and will not consider the pair-hopping term for simplicity. We evaluate the manyparticle Green's functions $\left[G_{a, \sigma}(\mathbf{k}, \omega)\right]$ of the MO Hamiltonian $H=H_{0}+H_{\text {int }}$ using the multiorbital iterated perturbation theory (MO-IPT) as impurity solver [42]. This interpolative ansatz is known to account for the correct low- and highenergy behavior of the one-particle spectral function and selfenergies of Hubbard and periodic Anderson like models in the large- $D$ limit (DMFT). It ensures the Mott-Hubbard metalinsulator transition from a correlated metal to a Mott insulator as a function of the Coulomb interaction $U$. The MO-IPT scheme is computationally very efficient, with real frequency output at zero and finite temperatures [43], enabling the study electronic structure reconstruction and transport properties of real materials with different magnetically ordered states and superconducting phase instabilities. The full set of equations for the MO case can be found in Ref. [42] so we do not repeat the equations here.

To elucidate the bandwidth-controlled Mott mechanism, in Fig. 5 we display the total LDA+DMFT DOS $\left[\rho_{\text {total }}(\omega)=\right.$ $\left.\sum_{a} \rho_{a}(\omega)\right]$ computed using $U=4.7 \mathrm{eV}$ and $J_{H}=0.7 \mathrm{eV}$ for the three regimes considered in this study: This particular $U$ was chosen since it is the critical value for opening the Mott bandgap at 7.0 GPa. As seen in this figure, at AP $\mathrm{BaFe}_{2} \mathrm{Se}_{3}$ is a Mott insulator, where all LDA bands split into two branches by sizable on-site Coulomb scattering of 


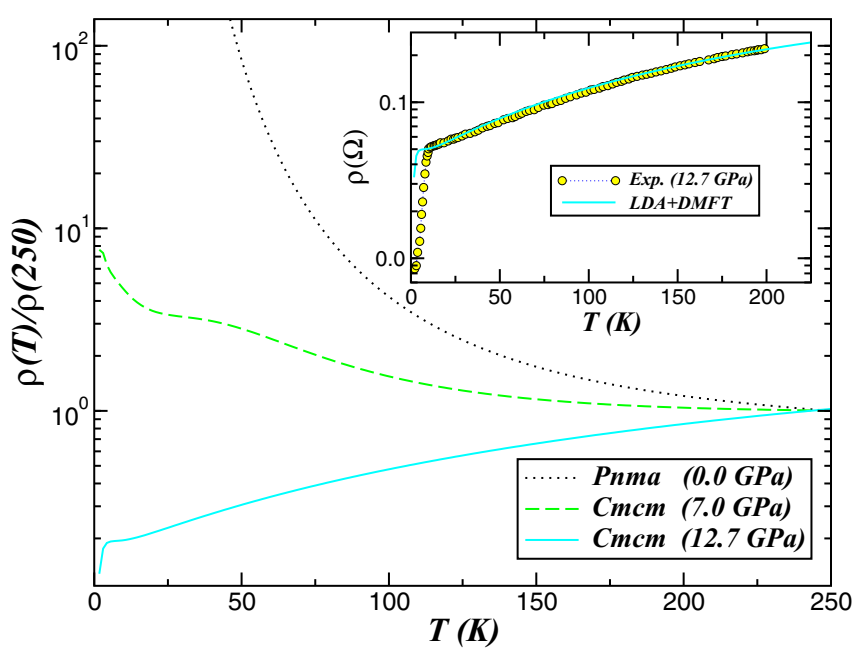

FIG. 6. $T$ dependence of electrical resistivity (normalized at $250 \mathrm{~K}$ ) for insulating and metallic $\mathrm{BaFe}_{2} \mathrm{Se}_{3}$, showing qualitative good accord with transport data [11] at different pressures. The semiconducting and metallic behavior for the compressed $(\mathrm{Cmcm})$ phase is consistent with normal state transport data. Inset: Theoryexperiment comparison of resistivity versus temperature normalized to the experimental value at $12.7 \mathrm{GPa}[11]$.

electrons within DMFT [17]. In this regime the one-particle spectral weight is transferred from low energies to lowerand upper-Hubbard bands at -1.5 and $3.5 \mathrm{eV}$, respectively. Hubbard bands are also visible in a similar energy window in the spectral function at 7.0 GPa. The valence band state in these two orbital-blocked Mott phases of $\mathrm{BaFe}_{2} \mathrm{Se}_{3}$ show similar tendency toward local moment formation, attesting the stability of the insulating phase at low-pressure conditions, as seen in Fig. 5.

Additionally, in Fig. 5 we compare our all-electron Mottinsulating state to photoemission data [23]. Good quantitative agreement over most of the energy scale from zero to $-1.2 \mathrm{eV}$ is observed. In addition to the detailed line shape at low binding energies, good agreement with the intense peak in UPS at $-1.0 \mathrm{eV}$ is visible, proving that LDA+DMFT(MO-IPT) is suitable to capture exotic electronic structure reconstruction phenomena in correlated electronic materials [26]. Particular relevant in this context are valence band states at $12.7 \mathrm{GPa}$, which are more severely reshaped due to strong intraand interorbital charge and orbital excitations intrinsic to $\mathrm{BaFe}_{2} \mathrm{Se}_{3}$ [2,44]. Across the Mott transition the incoherent Hubbard bands loses part of their spectral weight due to dynamical spectral weight transfer from high to low energies, with concomitant formation of OS quasiparticles [45] near $E_{F}$ as shown below. Therefore, large-scale changes in spectral weight transfer (SWT) characterizes the Mott transition in Cmcm $\mathrm{BaFe}_{2} \mathrm{Se}_{3}$ at high pressures. The spectral functions are predicted to be highly reshaped by external pressure and future spectroscopy and optical experiments could verify this aspect.

Figure 6 shows the $T$ dependence of the electrical resistivity $\rho_{d c}(T)$ of $\mathrm{BaFe}_{2} \mathrm{Se}_{3}$, computed using the MO spectral functions [46] at AP and the two pressurized regimes considered in Fig. 5. The insulating behavior is caused by strong

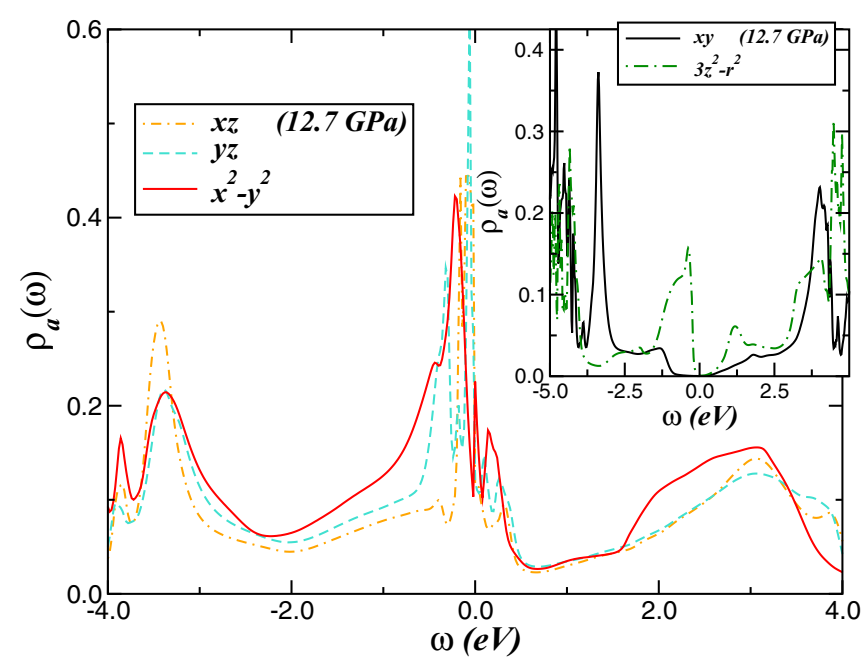

FIG. 7. Orbital-resolved LDA+DMFT DOS for the Fe $3 d$ orbitals of $\mathrm{BaFe}_{2} \mathrm{Se}_{3}$ at $12.7 \mathrm{GPa}$, showing coexistence of metallic (main panel) and Mott insulating (inset) states near $E_{F}$. Notice the low-energy Kondo-quasiparticle resonances and the incoherent Hubbard bands above $3.0 \mathrm{eV}$, a fingerprint of electron correlations effects in metals.

Coulomb repulsion between $\mathrm{Fe} 3 d$ electrons, which as in experiments $[11,12,15]$ becomes prominent at AP conditions. This orbital-blocked Mott state is partially suppressed within the $\mathrm{Cmcm}$ phase at $7.0 \mathrm{GPa}$. Noteworthy, due to partial increase of the bare bandwidth, the resistivity curve shows an insulating upturn below $18 \mathrm{~K}$ in good agreement with experiment [11]. Interestingly, the detailed $T$ dependence in Fig. 6 resembles the one seen in experiment both at ambient and moderated pressures, providing support for the correlated scenario derived above. Also remarkable is the change from the insulating to metallic behavior with increasing pressure from 7.0 to $12.7 \mathrm{GPa}$. Thus, reducing by effective $U / W$ ratio on increasing the one-particle bandwidth $W$ with pressure [17], we derive an OS metallic state which correctly describes the $T$ evolution of $\rho_{d c}(T)$ close to the superconducting phase instability, as shown in the inset of Fig. 6. Apart from the good theory-experiment agreement, a particularly interesting feature is the resistivity drop below $4 \mathrm{~K}$ also observed in experiments at $T \approx 9.5 \mathrm{~K}$ [11]. Taken together our results in Figs. 5 to 6 with extant spectroscopy [23] and transport experiments [11] confirms that metal-insulator transition in $\mathrm{BaFe}_{2} \mathrm{Se}_{3}$ can be characterized as a bandwidth-controlled Mott transition similar to what has been proposed for $\mathrm{BaFe}_{2} \mathrm{~S}_{3}[8]$.

To rationalize the overall metallic behavior and sudden resistivity decrease of pressurized $\mathrm{BaFe}_{2} \mathrm{Se}_{3}$ at low $T$ [11], in Fig. 7 we display the orbital resolved spectral functions used to compute the $T$ dependence of $\rho(T)$ at $12.7 \mathrm{GPa}$ in Fig. 6. In this pressure range metallic $\left(x z, y z, x^{2}-y^{2}\right)$ and localized $\left(x y, 3 z^{2}-r^{2}\right)$ electronic states coexist. This remarkable situation is closely tied to the OS Mott state [43,47,50], in which electronic localization is restricted to a particular subset of the active orbitals present in the problem.

Figure 7 can be taken as a microscopic evidence that localized and itinerant electrons coexist in Ba-123-spin ladder 
compounds [23]. Similarly to $\mathrm{BaFe}_{2} \mathrm{~S}_{3}$, where metallicity with $y z, x^{2}-y^{2}$ orbital character is predicted to exist near the allelectron Mott phase [48], asymmetric Kondo-quasiparticles with $x z, y z, x^{2}-y^{2}$ orbital character are predicted to coexist with Mott localized $x y, 3 z^{2}-r^{2}$ electronic states at low energies. Thus, apart from crystal field splitting effects [38,49], the threefold orbital character of the hidden metal is also relevant for the superconducting state of $\mathrm{Cmcm} \mathrm{BaFe}_{2} \mathrm{Se}_{3}$. Moreover, the presence of well-defined Kondo-quasiparticle resonances also suggests that double-exchange-like [50] charge and spin fluctuations between itinerant and localized channels are greatly suppressed, leading to an orbital decoupling phenomenon of strong correlations [45] at $12.7 \mathrm{GPa}$. Since infrared Fermi-liquid behavior is relatively immune to scattering between distinct $\mathrm{MO}$ components, this must be seen a manifestation that Anderson orthogonality catastrophe [51] is absent in the single crystals of Ref. [11]. According to our results a pressure-induced confined metal [19] with mostly $x z, y z, x^{2}-y^{2}$ orbital character could be observed in future experiments of compressed $\mathrm{BaFe}_{2} \mathrm{Se}_{3}$. The sudden decrease around $11 \mathrm{~K}$, which together with susceptibility signals was taken as fingerprint for the superconducting phase transition in $\mathrm{BaFe}_{2} \mathrm{Se}_{3}$ [11], is caused by the presence of a narrow Kondo-quasiparticle peak with $x^{2}-y^{2}$ orbital character at $E_{F}$. This is the key step, which allows to understand the low- $T$ drop in resistivity observed at pressure range between 11.5 to 12.7 GPa [11].

Since the correlated Fermi sea expected to be seen in future experiments at pressures close to $12.7 \mathrm{GPa}$ can be described in terms of well-defined quasiparticles, it is instructive to look at the orbital-resolved scattering rates $\Gamma_{a}(\omega)=$ $-2 Z_{a}(\omega) \operatorname{Im} \Sigma_{a}(\omega)$ [52] as a function of binding energy derived from $\mathrm{LDA}+\mathrm{DMFT}$ for $U=4.7 \mathrm{eV}$. Here

$$
Z_{a}(\omega)=\left[1-\frac{\partial \operatorname{Re} \Sigma_{a}(\omega)}{\partial \omega}\right]^{-1}
$$

is the quasiparticle residue, or the inverse of the electron mass renormalization, and $\operatorname{Re} \Sigma_{a}, \operatorname{Im} \Sigma_{a}$ are, respectively, the real and imaginary parts of electron's self-energy computed within the DMFT approximation. $Z$ characterizes the degree of electronic correlations of the normal metallic state, establishing the energy above which the lifetime of quasiparticles becomes short and many-particle coherence is lost [53].

Interestingly, our results for $\Gamma_{a}(\omega)$ in Fig. 8 suggest that compressed $\mathrm{BaFe}_{2} \mathrm{Se}_{3}$ is close to a Hund's metal state [45], where the interplay between $U, U^{\prime}$ and the Hund's exchange interaction $J_{H}$ leads to decoupled orbital degrees of freedom as proposed to $\mathrm{LiFeAs}$ superconductor [54]. In spite of the different Kondo-screening mechanisms similar lifetimes as found here have been extracted from angle-resolved photoemission (ARPES) linewidths of LiFeAs [55], indicating a common Hund's metal scenario in these systems. Also interesting is the energy dependence of the quasiparticle weights $Z_{a}(\omega)$, indicating OS correlation effects and dynamical mass enhancement $\left[m_{a}^{*} / m_{e}=Z_{a}^{-1}(0)\right]$ due to slightly different LDA bandwidths in the correlated electronic structure of compressed $\mathrm{BaFe}_{2} \mathrm{Se}_{3}$. Future experiments are called for to corroborate this prediction.

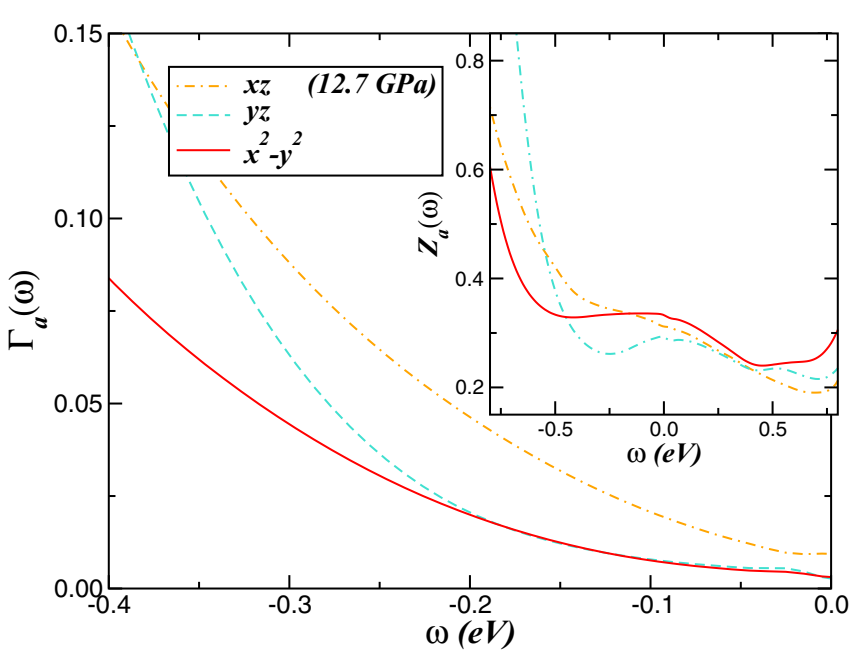

FIG. 8. Orbital-resolved scattering rates $\Gamma_{a}(\omega)(U=4.7 \mathrm{eV}$ and $J_{H}=0.7 \mathrm{eV}$ ) for the metallic orbitals of $\mathrm{BaFe}_{2} \mathrm{Se}_{3}$ at $12.7 \mathrm{GPa}$. Notice the small deviations from quadratic Fermi-liquid behavior due to residual interorbital scattering processes. The inset shows the corresponding quasiparticle weights or the inverse of the energy dependent mass renormalization factor.

\section{CONCLUSION}

In summary, we have used LDA+DMFT on a five-band Hubbard model to derive a pressure-induced electronic reconstruction in the spin-ladder compound $\mathrm{BaFe}_{2} \mathrm{Se}_{3}$. We have analyzed its Mott-insulating nature, baring it as an effect of multiorbital dynamical correlations. As a consequence of bandwidth broadening by pressure two distinct Mott insulating states are derived at ambient and moderate pressures. The metallic state, which is relevant to experiments at pressure close to superconducting phase instability [11], is shown to be of selective Mott type where localized and itinerant electrons coexist in the nonmagnetically ordered state. Particularly interesting is the emergence of low-energy Kondo-quasiparticles with $x z, y z, x^{2}-y^{2}$ orbital character in the selective metallic phase, which represents the mechanism for the normal-state electronic behavior seen in $\mathrm{BaFe}_{2} \mathrm{Se}_{3}$ at $12.7 \mathrm{GPa}$. The existence of well-defined quasiparticles suggests a Hund-induced orbital decoupling phenomenon [45] between Mott localized and itinerant components of the many-particle electronic fluid at high pressures. The interplay between local electronic correlations and bandwidth broadening in pressurized $\mathrm{BaFe}_{2} \mathrm{Se}_{3}$ yields a promising and practical route to access orbital-resolved quasiparticles [45] and scattering rates [56] of Hund's metals, but this prediction for $\mathrm{BaFe}_{2} \mathrm{Se}_{3}$ remains to be seen in future studies.

\section{ACKNOWLEDGMENTS}

L.C.'s work is supported by CNPq (Grant No. 304035/2017-3). Acknowledgment (L.C.) is also made to IFW Dresden for hospitality in earlier stages of this work and to CAPES. S.L. acknowledges support from the UK Research Council for using work in the paper that was undertaken by a student under Project No. EP/M50631X/1 as well as the DFG for support under the priority project SPP1415 and for a 
personal Heisenberg grant. S.L. also thanks ARCCA Cardiff for computational resources. Via S.L.'s membership of the UK's HPC Materials Chemistry Consortium, which is funded by EPSRC (No. EP/L000202), this work made use of the facilities of ARCHER, the UK's National High-Performance Computing Service, which is funded by the Office of Science and Technology through EPSRC's High End Computing Programme. We thank A. Yaresko for useful discussions.
[1] J. Rincón, A. Moreo, G. Alvarez, and E. Dagotto, Phys. Rev. B 90, 241105(R) (2014); T. Hajiri, T. Ito, M. Matsunami, B. H. Min, Y. S. Kwon, K. Kuroki, and S. Kimura, ibid. 93, 024503 (2016); D. Liu, C. Li, J. Huang, B. Lei, L. Wang, X. Wu, B. Shen, Q. Gao, Y. Zhang, X. Liu, Y. Hu, Y. Xu, A. Liang, J. Liu, P. Ai, L. Zhao, S. He, L. Yu, G. Liu, Y. Mao, X. Dong, X. Jia, F. Zhang, S. Zhang, F. Yang, Z. Wang, Q. Peng, Y. Shi, J. Hu, T. Xiang, X. Chen, Z. Xu, C. Chen, and X. J. Zhou, Phys. Rev. X 8, 031033 (2018); S.-H. Baek, D. Bhoi, W. Nam, B. Lee, D. V. Efremov, B. Büchner, and K. H. Kim, Nat. Commun. 9, 2139 (2018).

[2] K. Takubo, Y. Yokoyama, H. Wadati, S. Iwasaki, T. Mizokawa, T. Boyko, R. Sutarto, F. He, K. Hashizume, S. Imaizumi, T. Aoyama, Y. Imai, and K. Ohgushi, Phys. Rev. B 96, 115157 (2017).

[3] Y. Kamihara, T. Watanabe, M. Hirano, and H. Hosono, J. Am. Chem. Soc. 130, 3296 (2008).

[4] M. M. Qazilbash, J. J. Hamlin, R. E. Baumbach, L. Zhang, D. J. Singh, M. B. Maple, and D. N. Basov, Nat. Phys. 5, 647 (2009); M. Yi, Z.-K. Liu, Y. Zhang, R. Yu, J.-X. Zhu, J. J. Lee, R. G. Moore, F. T. Schmitt, W. Li, S. C. Riggs, J.-H. Chu, B. Lv, J. Hu, M. Hashimoto, S.-K. Mo, Z. Hussain, Z. Q. Mao, C. W. Chu, I. R. Fisher, Q. Si, Z.-X. Shen, and D. H. Lu, Nat. Commun. 6, 7777 (2015).

[5] P. Dai, Rev. Mod. Phys. 87, 855 (2015).

[6] I. I. Mazin, D. J. Singh, M. D. Johannes, and M. H. Du, Phys. Rev. Lett. 101, 057003 (2008); K. Terashima, Y. Sekiba, J. H. Bowen, K. Nakayama, T. Kawahara, T. Sato, P. Richard, Y.-M. Xu, L. J. Li, G. H. Cao, Z.-A. Xu, H. Ding, and T. Takahashi, Proc. Natl. Acad. Sci. USA 106, 7330 (2009); A. V. Chubukov, M. Khodas, and R. M. Fernandes, Phys. Rev. X 6, 041045 (2016)

[7] Y. J. Yan, M. Zhang, A. F. Wang, J. J. Ying, Z. Y. Li, W. Qin, X. G. Luo, J. Q. Li, J. Hu, and X. H. Chen, Sci. Rep. 2, 212 (2012); R. Yu, P. Goswami, Q. Si, P. Nikolic, and J.-X. Zhu, Nat. Commun. 4, 2783 (2013); C. C. Soares, M. ElMassalami, Y. Yanagisawa, M. Tanaka, H. Takeya, and Y. Takano, ibid. 8, 7041 (2018).

[8] H. Takahashi, A. Sugimoto, Y. Nambu, T. Yamauchi, Y. Hirata, T. Kawakami, M. Avdeev, K. Matsubayashi, F. Du, C. Kawashima, H. Soeda, S. Nakano, Y. Uwatoko, Y. Ueda, T. J. Sato, and K. Ohgushi, Nat. Mater. 14, 1008 (2015); T. Yamauchi, Y. Hirata, Y. Ueda, and K. Ohgushi, Phys. Rev. Lett. 115, 246402 (2015).

[9] M.-T. Suzuki, R. Arita, and H. Ikeda, Phys. Rev. B 92, 085116 (2015); L. Zheng, B. A. Frandsen, C. Wu, M. Yi, S. Wu, Q. Huang, E. Bourret-Courchesne, G. Simutis, R. Khasanov, D.-X. Yao, M. Wang, and R. J. Birgeneau, ibid. 98, 180402(R) (2018).

[10] N. D. Patel, A. Nocera, G. Alvarez, R. Arita, A. Moreo, and E. Dagotto, Phys. Rev. B 94, 075119 (2016).

[11] J. Ying, H. Lei, C. Petrovic, Y. Xiao, and V. V. Struzhkin, Phys. Rev. B 95, 241109(R) (2017).
[12] T. Hawai, C. Kawashima, K. Ohgushi, K. Matsubayashi, Y. Nambu, Y. Uwatoko, T. J. Sato, and H. Takahashi, J. Phys. Soc. Jpn. 86, 024701 (2017).

[13] V. Svitlyk, G. Garbarino, A. D. Rosa, E. Pomjakushina, A. Krzton-Maziopa, K. Conder, M. Nunez-Regueiro, and M. Mezouar, J. Phys.: Condens. Matter 31, 085401 (2019).

[14] H. Lei, H. Ryu, A. I. Frenkel, and C. Petrovic, Phys. Rev. B 84, 214511 (2011).

[15] F. Du, Y. Hirata, K. Matsubayashi, Y. Uwatoko, Y. Ueda, and K. Ohgushi, Phys. Rev. B 90, 085143 (2014).

[16] T. Hawai, Y. Nambu, K. Ohgushi, F. Du, Y. Hirata, M. Avdeev, Y. Uwatoko, Y. Sekine, H. Fukazawa, J. Ma, S. Chi, Y. Ueda, H. Yoshizawa, and T. J. Sato, Phys. Rev. B 91, 184416 (2015).

[17] M. Imada, A. Fujimori, and Y. Tokura, Rev. Mod. Phys. 70, 1039 (1998).

[18] H. Oike, K. Miyagawa, H. Taniguchi, and K. Kanoda, Phys. Rev. Lett. 114, 067002 (2015).

[19] Y. Ding, L. Yang, C.-C. Chen, H.-S. Kim, M. J. Han, W. Luo, Z. Feng, M. Upton, D. Casa, J. Kim, T. Gog, Z. Zeng, G. Cao, H.-K. Mao, and M. van Veenendaal, Phys. Rev. Lett. 116, 216402 (2016).

[20] J. M. Caron, J. R. Neilson, D. C. Miller, K. Arpino, A. Llobet, and T. M. McQueen, Phys. Rev. B 85, 180405(R) (2012).

[21] B. Saparov, S. Calder, B. Sipos, H. Cao, S. Chi, D. J. Singh, A. D. Christianson, M. D. Lumsden, and A. S. Sefat, Phys. Rev. B 84, 245132 (2011).

[22] M. Mourigal, S. Wu, M. B. Stone, J. R. Neilson, J. M. Caron, T. M. McQueen, and C. L. Broholm, Phys. Rev. Lett. 115, 047401 (2015).

[23] D. Ootsuki, N. L. Saini, F. Du, Y. Hirata, K. Ohgushi, Y. Ueda, and T. Mizokawa, Phys. Rev. B 91, 014505 (2015).

[24] J. Herbrych, N. Kaushal, A. Nocera, G. Alvarez, A. Moreo, and E. Dagotto, Nat. Commun. 9, 3736 (2018).

[25] V. Svitlyk, D. Chernyshov, E. Pomjakushina, A. KrztonMaziopa, K. Conder, V. Pomjakushin, R. Pottgen, and V. Dmitriev, J. Phys.: Condens. Matter 25, 315403 (2013).

[26] G. Kotliar, S. Y. Savrasov, K. Haule, V. S. Oudovenko, O. Parcollet, and C. A. Marianetti, Rev. Mod. Phys. 78, 865 (2006).

[27] A. Krzton-Maziopa, E. Pomjakushina, V. Pomjakushin, D. Sheptyakov, D. Chernyshov, V. Svitlyk, and K. Conder, J. Phys.: Condens. Matter 23, 402201 (2011).

[28] O. K. Andersen, Phys. Rev. B 12, 3060 (1975).

[29] Y. Zhang, L.-F. Lin, J.-J. Zhang, E. Dagotto, and S. Dong, Phys. Rev. B 97, 045119 (2018).

[30] M. V. Medvedev, I. A. Nekrasov, and M. V. Sadovskii, JETP Lett. 95, 33 (2012).

[31] J. Kuneš, in The LDA+DMFT Approach to Strongly Correlated Materials, Modeling and Simulation, edited by E. Pavarini, E. Koch, D. Vollhardt, and A. Lichtenstein (Verlag des Forschungszentrum Jülich, Jülich, 2011), Vol. 1. 
[32] N. Marzari, A. A. Mostofi, J. R. Yates, I. Souza, and D. Vanderbilt, Rev. Mod. Phys. 84, 1419 (2012).

[33] P. Giannozzi et al., J. Phys.: Condens. Matter 21, 395502 (2009).

[34] A. Dal Corso, Comput. Mater. Sci. 95, 337 (2014).

[35] Y. Zhang, L.-F. Lin, A. Moreo, S. Dong, and E. Dagotto, Phys. Rev. B 101, 144417 (2020).

[36] Y. Zhang, L.-F. Lin, A. Moreo, S. Dong, and E. Dagotto, Phys. Rev. B 100, 184419 (2019).

[37] L. Craco, M. S. Laad, S. Leoni, and E. Müller-Hartmann, Phys. Rev. B 70, 195116 (2004).

[38] S. Chi, Y. Uwatoko, H. Cao, Y. Hirata, K. Hashizume, T. Aoyama, and K. Ohgushi, Phys. Rev. Lett. 117, 047003 (2016).

[39] L. Craco, T. A. da Silva Pereira, S. R. Ferreira, S. S. Carara, and S. Leoni, Sci. Rep. 7, 2632 (2017), and references therein. L. Craco et al., Phys. Rev. B 98, 035114 (2018).

[40] L. Craco and S. Leoni, Sci. Rep. 7, 46439 (2017), and references therein.

[41] K. Held and D. Vollhardt, Eur. Phys. J. B 5, 473 (1998); R. Yu, J.-X. Zhu, and Q. Si, Phys. Rev. Lett. 106, 186401 (2011); G. Liu, N. Kaushal, S. Li, C. B. Bishop, Y. Wang, S. Johnston, G. Alvarez, A. Moreo, and E. Dagotto, Phys. Rev. E 93, 063313 (2016); L. Craco, Phys. Rev. B 101, 085131 (2020).

[42] L. Craco, Phys. Rev. B 77, 125122 (2008).

[43] M. S. Laad, L. Craco, and E. Müller-Hartmann, Phys. Rev. B 73, 045109 (2006).

[44] N. D. Patel, A. Nocera, G. Alvarez, A. Moreo, S. Johnston, and E. Dagotto, Nat. Commun. 2, 64 (2019).
[45] A. Kostin, P. O. Sprau, A. Kreisel, Yi Xue Chong, A. E. Böhmer, P. C. Canfield, P. J. Hirschfeld, B. M. Andersen, and J. C. Sámus Davis, Nat. Mater. 17, 869 (2018).

[46] K. Haule and G. Kotliar, New J. Phys. 11, 025021 (2009).

[47] A. Liebsch, Phys. Rev. B 70, 165103 (2004); L. de'Medici S. R. Hassan, M. Capone, and X. Dai, Phys. Rev. Lett. 102, 126401 (2009).

[48] L. Craco and S. Leoni, Phys. Rev. B 98, 195107 (2018).

[49] J. Rincón, A. Moreo, G. Alvarez, and E. Dagotto, Phys. Rev. Lett. 112, 106405 (2014).

[50] S. Biermann, L. de Medici, and A. Georges, Phys. Rev. Lett. 95, 206401 (2005).

[51] P. W. Anderson, The Theory of Superconductivity in the High$\mathrm{T}_{c}$ Cuprates, Princeton Series in Physics (Princeton University Press, Princeton, NJ, 1997).

[52] J. Fink, J. Nayak, E. D. L. Rienks, J. Bannies, S. Wurmehl, S. Aswartham, I. Morozov, R. Kappenberger, M. A. ElGhazali, L. Craco, H. Rosner, C. Felser, and B. Büchner, Phys. Rev. B 99, 245156 (2019).

[53] N. Trivedi, Nat. Phys. 4, 163 (2008).

[54] H. Miao, Z. P. Yin, S. F. Wu, J. M. Li, J. Ma, B.-Q. Lv, X. P. Wang, T. Qian, P. Richard, L.-Y. Xing, X.-C. Wang, C. Q. Jin, K. Haule, G. Kotliar, and H. Ding, Phys. Rev. B 94, 201109(R) (2016).

[55] V. Brouet, D. LeBoeuf, P.-H. Lin, J. Mansart, A. Taleb-Ibrahimi, P. Le Fèvre, F. Bertran, A. Forget, and D. Colson, Phys. Rev. B 93, 085137 (2016).

[56] J. Nayak, K. Filsinger, G. H. Fecher, S. Chadov, J. Minár, E. D. L. Rienks, B. Büchner, S. P. Parkin, J. Fink, and C. Felser, Proc. Natl. Acad. Sci. USA 114, 12425 (2017). 